\title{
Ideal convergence of double sequences in random 2-normed spaces
}

\author{
Syed Abdul Mohiuddine*, Abdullah Alotaibi and Saud M Alsulami
}

${ }^{*}$ Correspondence:

mohiuddine@gmail.com

Department of Mathematics,

Faculty of Science, King Abdulaziz

University, P.O. Box 80203, Jeddah,

21589, Saudi Arabia

\begin{abstract}
Quite recently, Alotaibi and Mohiuddine (Adv. Differ. Equ. 2012:39, 2012) studied the idea of a random 2-normed space to determine some stability results concerning the cubic functional equation. In this paper, we define and study the concepts of $\mathcal{I}$-convergence and $\mathcal{I}^{*}$-convergence for double sequences in random 2 -normed spaces and establish the relationship between these types of convergence, i.e., we show that $\mathcal{I}^{*}$-convergence implies $\mathcal{I}$-convergence in random 2 -normed spaces. Furthermore, we have also demonstrated through an example that, in general, $\mathcal{I}$-convergence does not imply $\mathcal{I}^{*}$-convergence in random 2-normed spaces. MSC: $40 \mathrm{~A} 05 ; 46 \mathrm{~A} 70$
\end{abstract}

Keywords: double sequences; $t$-norm; random 2-normed spaces; ideal convergence

\section{Introduction}

Menger [1] generalized the metric axioms by associating a distribution function with each pair of points of a set. This system, called a probabilistic metric space, originally a statistical metric space, has been developed extensively by Schweizer and Sklar [2]. The idea of Menger was to use distribution functions instead of nonnegative real numbers. An important family of probabilistic metric spaces are probabilistic normed spaces. Probabilistic normed spaces are real linear spaces in which the norm of each vector is an appropriate probability distribution function rather than a number. Such spaces were first introduced by Šerstnev in 1963 [3]. In [4], Alsina et al. gave a new definition of PN spaces that includes Šerstnev's and leads naturally to the identification of the principal class of PN spaces, the Menger spaces. Recently, the concept of probabilistic normed spaces was extended to random/probabilistic 2-normed spaces by Golet [5] using the concept of 2-norm of Gähler [6].

The concept of statistical convergence for sequences of real numbers was introduced by Fast [7] in 1951, and since then several generalizations and applications of this notion have been investigated by various authors (see [8-21]). The notion of statistical convergence is a very useful functional tool for studying the convergence problems of numerical sequences/matrices (double sequences) through the concept of density. The concept of $I$-convergence, which is a generalization of statistical convergence, was introduced by Kastyrko, Salat and Wilczynski [22] by using the ideal $I$ of subsets of the set of natural numbers $\mathbb{N}$ and further studied in [23-27] and references therein. Recently, Şahiner et al. [28], and Gürdal and Acik [29] studied ideal convergence and $I$-Cauchy sequences respectively in 2-normed spaces, and also Gürdal and Pehlivan [30] studied statistical convergence in 
a 2-Banach space. Most recently, Alotaibi and Mohiuddine [31] determined the stability of a cubic functional equation in a random 2-normed space.

In this paper, we study the concept of $\mathcal{I}$-convergence and $\mathcal{I}^{\prime \prime}$-convergence in a more general setting, i.e., in a random 2-normed space. We discuss the relationship between $\mathcal{I}$-convergence and $\mathcal{I}^{\prime \prime}$-convergence and prove some interesting results.

\section{Definitions, notations and preliminary results}

In this section, we recall some basic definitions and notations which form the background of the present work.

A distribution function is an element of $\Delta^{+}$, where $\Delta^{+}=\{f: \mathbb{R} \rightarrow[0,1] ; f$ is leftcontinuous, nondecreasing, $f(0)=0$ and $f(+\infty)=1\}$, and the subset $D^{+} \subseteq \Delta^{+}$is the set $D^{+}=\left\{f \in \Delta^{+} ; l^{-} f(+\infty)=1\right\}$. Here $l^{-} f(+\infty)$ denotes the left limit of the function $f$ at the point $x$. The space $\Delta^{+}$is partially ordered by the usual pointwise ordering of functions, i.e., $f \leq g$ if and only if $f(x) \leq g(x)$ for all $x \in \mathbb{R}$. For any $a \in \mathbb{R}, \varepsilon_{a}$ is a distribution function defined by

$$
\varepsilon_{a}(x)= \begin{cases}0 & \text { if } x \leq a \\ 1 & \text { if } x>a\end{cases}
$$

The set $\Delta$, as well as its subsets, can be partially ordered by the usual pointwise order: in this order, $\varepsilon_{0}$ is the maximal element in $\Delta^{+}$.

A triangle function is a binary operation on $\Delta^{+}$, namely a function $\tau: \Delta^{+} \times \Delta^{+} \rightarrow \Delta^{+}$, that is associative, commutative, nondecreasing, and has $\varepsilon_{0}$ as unit; that is, for all $f, g, h \in$ $\Delta^{+}$, we have:

(i) $\tau(\tau(f, g), h)=\tau(f, \tau(g, h))$,

(ii) $\tau(f, g)=\tau(g, f)$,

(iii) $\tau(f, g)=\tau(g, f)$ whenever $f \leq g$,

(iv) $\tau\left(f, \varepsilon_{0}\right)=f$.

A $t$-norm is a binary operation $*:[0,1] \times[0,1] \rightarrow[0,1]$ such that for all $a, b, c, d \in[0,1]$ we have:

(i) $*$ is associative and commutative, (ii) $*$ is continuous, (iii) $a * 1=a$, (iv) $a * b \leq c * d$ whenever $a \leq c$ and $b \leq d$.

The concept of a 2-normed space was first introduced by Gähler [6].

Let $X$ is a linear space of a dimension $d$, where $2 \leq d<\infty$. A 2-norm on $X$ is a function $\|\cdot, \cdot\|: X \times X \rightarrow \mathbb{R}$ satisfying the following conditions: for every $x, y \in X$, (i) $\|x, y\|=0$ if and only if $x$ and $y$ are linearly dependent; (ii) $\|x, y\|=\|y, x\|$; (iii) $\|\alpha x, y\|=|\alpha|\|x, y\|, \alpha \in \mathbb{R}$; (iv) $\|x+y, z\| \leq\|x, z\|+\|y, z\|$. In this case $(X,\|\cdot, \cdot\|)$ is called a 2-normed space.

Example 2.1 Take $X=\mathbb{R}^{2}$ being equipped with the 2-norm $\|x, y\|=$ the area of the parallelogram spanned by the vectors $x$ and $y$, which may be given explicitly by the formula

$$
\|x, y\|=\left|x_{1} y_{2}-x_{2} y_{1}\right|, \quad \text { where } x=\left(x_{1}, x_{2}\right), y=\left(y_{1}, y_{2}\right) .
$$

Recently, Goleț [5] introduced the notion of a random 2-normed space as follows.

Let $X$ be a linear space of a dimension greater than one, $\tau$ a triangle function, and $\mathcal{F}$ : $X \times X \rightarrow \Delta^{+}$. Then $\mathcal{F}$ is called a probabilistic 2-norm on $X$ and $(X, \mathcal{F}, \tau)$ a probabilistic 2-normed space if the following conditions are satisfied: 
(i) $\mathcal{F}_{x, y}(t)=\varepsilon_{0}(t)$ if $x$ and $y$ are linearly dependent, where $\mathcal{F}_{x, y}(t)$ denotes the value of $\mathcal{F}_{x, y}$ at $t \in \mathbb{R}$,

(ii) $\mathcal{F}_{x, y} \neq \varepsilon_{0}$ if $x$ and $y$ are linearly independent,

(iii) $\mathcal{F}_{x, y}=\mathcal{F}_{y, x}$ for every $x, y$ in $X$,

(iv) $\mathcal{F}_{\alpha x, y}(t)=\mathcal{F}_{x, y}\left(\frac{t}{|\alpha|}\right)$ for every $t>0, \alpha \neq 0$ and $x, y \in X$,

(v) $\mathcal{F}_{x+y, z} \geq \tau\left(\mathcal{F}_{x z}, \mathcal{F}_{y z}\right)$ whenever $x, y, z \in X$.

If $(\mathrm{v})$ is replaced by

$\left(\mathrm{v}^{\prime}\right) \mathcal{F}_{x+y, z}\left(t_{1}+t_{2}\right) \geq \mathcal{F}_{x, z}\left(t_{1}\right) * \mathcal{F}_{y, z}\left(t_{2}\right)$, for all $x, y, z \in X$ and $t_{1}, t_{2} \in \mathbb{R}_{0}^{+}$,

then triple $(X, \mathcal{F}, *)$ is called a random 2-normed space (for short, RTN-space).

Example 2.2 Let $(X,\|\cdot, \cdot\|)$ be a 2 -normed space with $\|x, z\|=\left\|x_{1} z_{2}-x_{2} z_{1}\right\|, x=\left(x_{1}, x_{2}\right)$, $z=\left(z_{1}, z_{2}\right)$ and $a * b=a b$ for $a, b \in[0,1]$. For all $x \in X, t>0$ and nonzero $z \in X$, consider

$$
\mathcal{F}_{x, z}(t)= \begin{cases}\frac{t}{t+\|x, z\|} & \text { if } t>0, \\ 0 & \text { if } t \leq 0\end{cases}
$$

Then $(X, \mathcal{F}, *)$ is a random 2 -normed space.

Remark 2.3 Note that every 2-normed space $(X,\|\cdot, \cdot\|)$ can be made a random 2-normed space in a natural way, by setting $\mathcal{F}_{x, y}(t)=\varepsilon_{0}(t-\|x, y\|)$, for every $x, y \in X, t>0$ and $a * b=$ $\min \{a, b\}, a, b \in[0,1]$.

In 1900, Pringsheim [32] introduced the notion of convergence of double sequences as follows: A double sequence $x=\left(x_{j k}\right)$ is said to converge to the limit L in Pringsheim's sense (shortly, $P$-convergent to $L$ ) if for every $\varepsilon>0$ there exists an integer $N$ such that $\left|x_{j k}-L\right|<\varepsilon$ whenever $j, k>N$. In this case $L$ is called the $P$-limit of $x$.

Let $K \subseteq \mathbb{N} \times \mathbb{N}$ be a two-dimensional set of positive integers and let $K_{m, n}=\{(j, k): j \leq$ $m, k \leq n\}$. Then the two-dimensional analogue of natural density can be defined as follows.

In case the sequence $(K(m, n) / m n)$ has a limit in Pringsheim's sense, then we say that $K$ has a double natural density and is defined as

$$
P-\lim _{m, n} \frac{K(m, n)}{m n}=\delta_{2}(K)
$$

For example, let $K=\left\{\left(i^{2}, j^{2}\right): i, j \in \mathbb{N}\right\}$. Then

$$
\delta_{2}(K)=P-\lim _{m, n} \frac{K(m, n)}{m n} \leq P-\lim _{m, n} \frac{\sqrt{m} \sqrt{n}}{m n}=0
$$

i.e., the set $K$ has double natural density zero, while the set $\{(i, 2 j): i, j \in \mathbb{N}\}$ has double natural density $\frac{1}{2}$.

Statistical convergence for double sequences $x=\left(x_{j k}\right)$ of real numbers was introduced and studied by Mursaleen and Edely [17] and in intuitionistic fuzzy normed spaces by Mursaleen and Mohiuddine [18]. 
A real double sequence $x=\left(x_{j k}\right)$ is said to be statistically convergent to the number $\ell$ if for each $\epsilon>0$ the set

$$
\left\{(j, k), j \leq m \text { and } k \leq n:\left|x_{j k}-\ell\right| \geq \epsilon\right\}
$$

has double natural density zero. In this case we write $s t_{2}-\lim _{j, k} x_{j k}=\ell$ and denote the set of all statistically convergent double sequences.

If $X$ is a non-empty set, then a family of subsets of $X$ is called an ideal in $X$ if and only if

(a) $\emptyset \in I$,

(b) $A, B \in I$ implies $A \cup B \in I$,

(c) for each $A \in I$ and $B \subset A$ we have $B \in I$,

$I$ is called a nontrivial ideal if $X \in I \neq \emptyset$ and $P(X)$ is the power set of $X$.

Let $X$ be a non-empty set. A non-empty family of sets $F \subset P(X)$ is called a filter on $X$ if and only if

(a) $\emptyset \notin F$,

(b) $A, B \in F$ implies $A \cap B \in F$,

(c) for each $A \in F$ and $B \supset A$ we have $B \in F$.

A nontrivial ideal $I$ in $X$ is called an admissible ideal if it is different from $P(\mathbb{N})$ and contains all singletons, i.e., $\{x\} \in I$ for each $x \in X$.

Let $I \subset P(X)$ be a nontrivial ideal. Then a class $F(I)=\{M \subset X: M=X \backslash A$, for some $A \in$ $I\}$ is a filter on $X$, called the filter associated with the ideal $I$.

An admissible ideal $I \subset P(\mathbb{N})$ is said to satisfy the condition (AP) if for every sequence $\left(A_{n}\right)_{n \in \mathbb{N}}$ of pairwise disjoint sets from $I$ there are sets $B_{n} \subset \mathbb{N}, n \in \mathbb{N}$ such that the symmetric difference $A_{n} \triangle B_{n}$ is a finite set for every $n$ and $\bigcup_{n \in \mathbb{N}} B_{n} \in I$.

Let $I$ be a nontrivial ideal of $\mathbb{N}$. A sequence $x=\left(x_{k}\right)$ is said to be $I$-convergent [22] to $L \in X$ if, for each $\epsilon>0$, the set $\left\{k \in \mathbb{N}:\left|x_{k}-\ell\right| \geq \epsilon\right\} \in I$. In this case we write $I$ - $\lim x=L$.

\section{Main results}

In this section, we study the concept of $\mathcal{I}$-convergence and $\mathcal{I}^{*}$-convergence of double sequences in a random 2-normed space. We shall assume throughout this paper that $\mathcal{I}$ as a nontrivial ideal in $\mathbb{N} \times \mathbb{N}$ and $(X, \mathcal{F}, *)$ is a random 2-normed space.

We define:

Definition 3.1 A double sequence $x=\left(x_{j k}\right)$ is convergent in $(X, \mathcal{F}, *)$ or simply $(\mathcal{F})$ convergent to $\ell$ if, for every $\epsilon>0, \theta \in(0,1)$, there exists a positive integer $N$ such that $\mathcal{F}_{x_{j k}-\ell, z}(\epsilon)>1-\theta$ whenever $j, k \geq N$ and nonzero $z \in X$. In this case we write $(\mathcal{F})-\lim _{j, k \rightarrow \infty} x_{j k}=\ell$ and $\ell$ is called the $\mathcal{F}$-limit of $x=\left(x_{j k}\right)$.

Definition 3.2 Let $\mathcal{I}$ a be nontrivial ideal of $\mathbb{N} \times \mathbb{N}$. A double sequence $x=\left(x_{j k}\right)$ is said to

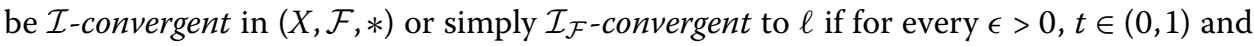
nonzero $z \in X$, we have $A(\epsilon, t) \in \mathcal{I}$, where

$$
A(\epsilon, t)=\left\{(j, k) \in \mathbb{N} \times \mathbb{N}: \mathcal{F}_{x_{j k}-\ell, z}(\epsilon) \leq 1-t\right\} .
$$

In this case we write $\mathcal{I}_{\mathcal{F}}-\lim x=\xi$. 
Definition 3.3 We say that a double sequence $x=\left(x_{j k}\right)$ is said to be $\mathcal{I}^{\prime \prime}$-convergent in $(X, \mathcal{F}, *)$ or simply $\mathcal{I}_{\mathcal{F}}^{*}$-convergent to $\ell$ if there exists a subset $K=\left\{\left(j_{m}, k_{m}\right): j_{1}<j_{2}<\cdots ; k_{1}<\right.$ $\left.k_{2}<\cdots\right\}$ of $\mathbb{N} \times \mathbb{N}$ such that $K \in F(\mathcal{I})($ i.e., $\mathbb{N} \times \mathbb{N} \backslash K \in \mathcal{I})$ and $\mathcal{F}$ - $\lim _{m} x_{j_{m} k_{m}}=\ell$. In this case we write $\mathcal{I}_{\mathcal{F}}^{*}-\lim x=\ell$ and $\ell$ is called the $\mathcal{I}_{\mathcal{F}}^{*}$-limit of the double sequence $x=\left(x_{j k}\right)$.

Theorem 3.4 If a double sequence $x=\left(x_{j k}\right)$ is $\mathcal{I}_{\mathcal{F}}$-convergent, then $\mathcal{I}_{\mathcal{F}}$-limit is unique.

Proof Suppose that $\mathcal{I}_{\mathcal{F}}-\lim x=\ell_{1}$ and $\mathcal{I}_{\mathcal{F}}-\lim x=\ell_{2}$. Given $\epsilon>0$, choose $s>0$ such that $(1-s) *(1-s)>1-\epsilon$. Then, for any $t>0$ and nonzero $z \in X$, define the following sets as:

$$
\begin{aligned}
& \mathcal{D}_{\mathcal{F}}^{\prime}(s, t)=\left\{(j, k) \in \mathbb{N} \times \mathbb{N}: \mathcal{F}_{x_{j k}-\ell_{1}, z}(t) \leq 1-s\right\}, \\
& \mathcal{D}_{\mathcal{F}}^{\prime \prime}(s, t)=\left\{(j, k) \in \mathbb{N} \times \mathbb{N}: \mathcal{F}_{x_{j k}-\ell_{2}, z}(t) \leq 1-s\right\} .
\end{aligned}
$$

Since $\mathcal{I}_{\mathcal{F}}-\lim x=\ell_{1}$, we have $\mathcal{D}_{\mathcal{F}}^{\prime}(s, t) \in \mathcal{I}$. Similarly $\mathcal{I}_{\mathcal{F}}-\lim x=\ell_{2}$ implies that $\mathcal{D}_{\mathcal{F}}^{\prime \prime}(s, t) \in \mathcal{I}$. Now let $\mathcal{D}_{\mathcal{F}}(s, t)=\mathcal{D}_{\mathcal{F}}^{\prime}(s, t) \cup \mathcal{D}_{\mathcal{F}}^{\prime \prime}(s, t)$. Then from the definition of $\mathcal{I}, \mathcal{D}_{\mathcal{F}}(s, t) \in \mathcal{I}$, and hence its compliment $\mathcal{D}_{\mathcal{F}}^{C}(s, t)$ is a non-empty set which belongs to $F(\mathcal{I})$. Now, if $(j, k) \in \mathbb{N} \times \mathbb{N} \backslash \mathcal{D}_{\mathcal{F}}(s, t)$, then we have

$$
\mathcal{F}_{\ell_{1}-\ell_{2}, z}(t) \geq \mathcal{F}_{x_{j k}-\ell_{1}, z}(t / 2) * \mathcal{F}_{x_{j k}-\ell_{2}, z}(t / 2)>(1-s) *(1-s)>1-\epsilon .
$$

Since $\epsilon>0$ was arbitrary, we get $\mathcal{F}_{\ell_{1}-\ell_{2}, z}(t)=1$ for all $t>0$ and nonzero $z \in X$. Hence $\ell_{1}=\ell_{2}$.

This completes the proof of the theorem.

Theorem 3.5 Let $\mathcal{I}$ be an admissible ideal. If a double sequence $x=\left(x_{j k}\right)$ is $(\mathcal{F})$-convergent to $\ell$, then it is $\mathcal{I}_{\mathcal{F}}$-convergent to the same limit. But the converse need not be true.

Proof Let $(\mathcal{F})-\lim x=\ell$. Then for every $\epsilon>0, t>0$ and nonzero $z \in X$, there is a positive integer $N$ such that

$$
\mathcal{F}_{x_{j k}-\ell, z}(t)>1-\epsilon
$$

for all $j, k \geq N$. Since the set

$$
A(\epsilon):=\left\{(j, k) \in \mathbb{N} \times \mathbb{N}: \mathcal{F}_{x_{j k}-\ell, z}(\epsilon) \leq 1-\epsilon\right\}
$$

is contained in $S \times S$, where $S=\{1,2,3, \ldots, N-1\}$ and the ideal $\mathcal{I}$ is admissible, $A(\epsilon) \in \mathcal{I}$. Hence $\mathcal{I}_{\mathcal{F}}-\lim x=\ell$.

For the converse, we construct the following example.

Example 3.6 Let $X=\mathbb{R}^{2}$, with the 2-norm $\|x, z\|=\left|x_{1} z_{2}-x_{2} z_{1}\right|, x=\left(x_{1}, x_{2}\right), z=\left(z_{1}, z_{2}\right)$, and $a * b=a b$ for all $a, b \in[0,1]$. Let $\mathcal{F}_{x, z}(t)=\frac{t}{t+\|x, z\|}$ for all $x, y \in X, t>0$. Now we define a double sequence $x=\left(x_{j k}\right)$ by

$$
x_{j k}= \begin{cases}(j k, 0) & \text { if } j \text { and } k \text { are squares; } \\ (0,0) & \text { otherwise. }\end{cases}
$$


Write

$$
K_{m, n}(\epsilon, t):=\left\{j \leq m, k \leq n: \mathcal{F}_{x_{j k}-\ell, z}(t) \leq 1-\epsilon\right\}, \quad 0<\epsilon<1 ; \ell=(0,0) .
$$

We see that

$$
\mathcal{F}_{x_{j k}-\ell, z}(t)= \begin{cases}\frac{t}{t+j k z 2} & \text { if } j \text { and } k \text { are squares; } \\ 1 & \text { otherwise. }\end{cases}
$$

Taking limit $j, k \rightarrow \infty$, we get

$$
\lim _{j, k \rightarrow \infty} \mathcal{F}_{x_{j k}-\ell, z}(t)= \begin{cases}0 & \text { if } j \text { and } k \text { are squares } \\ 1 & \text { otherwise }\end{cases}
$$

Hence, a double sequence $x=\left(x_{j k}\right)$ is not convergent in $(X, \mathcal{F}, *)$. But if we take $\mathcal{I}=$ $\mathcal{I}(\delta)=\left\{A \subset \mathbb{N} \times \mathbb{N}: \delta_{2}(A)=0\right\}$, then since $K_{m, n}(\epsilon, t) \subset\{(1,0),(4,0),(9,0),(16,0), \ldots\}$, $\delta_{2}\left(K_{m, n}(\epsilon, t)\right)=0$, that is, $\mathcal{I}_{\mathcal{F}}-\lim x=\ell$.

This completes the proof of the theorem.

Theorem 3.7 Let $\mathcal{I}$ be an admissible ideal and $x=\left(x_{j k}\right)$ be a double sequence. If $\mathcal{I}_{\mathcal{F}}^{*}-\lim x=$ $\ell$ then $\mathcal{I}_{\mathcal{F}}-\lim x=\ell$.

Proof Suppose that $\mathcal{I}_{\mathcal{F}}^{*} \lim x=\ell$. Then there exists a subset $K=\left\{\left(j_{m}, k_{m}\right): j_{1}<j_{2}<\cdots ; k_{1}<\right.$ $\left.k_{2}<\cdots\right\}$ of $\mathbb{N} \times \mathbb{N}$ such that $K \in F(\mathcal{I})$ (i.e., $\mathbb{N} \times \mathbb{N} \backslash K \in \mathcal{I}$ ) and $\mathcal{F}-\lim _{m} x_{j_{m} k_{m}}=\ell$. But, for each $\epsilon>0$ and $t>0$ there exists $N \in \mathbb{N}$ such that $\mathcal{F}_{x_{j m} k_{m}-\ell, z}(t)>1-\epsilon$ for all $m>N$. Since $\left\{\left(j_{m}, k_{m}\right) \in K: \mathcal{F}_{x_{j_{m} k_{m}}-\ell, z}(t) \leq 1-\epsilon\right\}$ is contained in $\left\{j_{1}<j_{2}<\cdots<j_{N-1}\right\} \cup\left\{k_{1}<k_{2}<\cdots<\right.$ $\left.k_{N-1}\right\}$ and the ideal $\mathcal{I}$ is admissible, we have

$$
\left\{\left(j_{m}, k_{m}\right) \in K: \mathcal{F}_{x_{j_{m} k_{m}}-\ell, z}(t) \leq 1-\epsilon\right\} \in \mathcal{I}
$$

Thus

$$
\left\{(j, k) \in: \mathcal{F}_{x_{j k}-\ell, z}(t) \leq 1-\epsilon\right\} \subseteq H \cup\left\{j_{1}<j_{2}<\cdots<j_{N-1}\right\} \cup\left\{k_{1}<k_{2}<\cdots<k_{N-1}\right\} \in \mathcal{I}
$$

for all $\epsilon>0$ and $t>0$. Therefore, we conclude that $\mathcal{I}_{\mathcal{F}}-\lim x=\ell$.

This completes the proof of the theorem.

The following example shows that the converse of the above theorem need not be true.

Example 3.8 Let $X=\mathbb{R}^{2}$, with the 2-norm $\|x, z\|=\left|x_{1} z_{2}-x_{2} z_{1}\right|, x=\left(x_{1}, x_{2}\right), z=\left(z_{1}, z_{2}\right)$, and $a * b=a b$ for all $a, b \in[0,1]$. Define $\mathcal{F}_{x, z}(t)=\frac{t}{t+\|x, z\|}$ for all $x, y \in X$ and $t>0$. Then $(X, \mathcal{F}, *)$ is a RTN-space. Let $\mathbb{N} \times \mathbb{N}=\bigcup_{i, j} \triangle_{i j}$ be a decomposition of $\mathbb{N} \times \mathbb{N}$ such that, for any $(m, n) \in \mathbb{N} \times \mathbb{N}$ each $\triangle_{i j}$ contains infinitely many $(i, j)$ 's, where $i \geq m, j \geq n$ and $\triangle_{i j} \cap \triangle_{m n}=\emptyset$ for $(i, j) \neq(m, n)$. Now we define a double sequence $x=\left(x_{m n}\right)$ by $x_{m n}=\left(\frac{1}{i j}, 0\right)$ if $(m, n) \in \triangle_{i j}$. Then $\mathcal{F}_{x_{m n}, z}(t) \longrightarrow 1$ as $m, n \rightarrow \infty$. Hence $\mathcal{I}_{\mathcal{F}}-\lim _{m, n} x_{m n}=0$. 
On the other hand, suppose that $\mathcal{I}_{\mathcal{F}}^{*} \lim _{m, n} x_{m n}=0$. Then there exists a subset $K=$ $\left\{\left(m_{j}, n_{j}\right): m_{1}<m_{2}<\cdots ; n_{1}<n_{2}<\cdots\right\}$ of $\mathbb{N} \times \mathbb{N}$ such that $K \in F(\mathcal{I})$ and $\mathcal{F}-\lim _{j} x_{m_{j} n_{j}}=0$. Since $K \in F(\mathcal{I})$, there is a set $H \in \mathcal{I}$ such that $K=\mathbb{N} \times \mathbb{N} \backslash H$. Now, from the definition of $\mathcal{I}$, there exists say $p, q \in \mathbb{N}$ such that

$$
H \subset\left(\bigcup_{m=1}^{p}\left(\bigcup_{n=1}^{\infty} \triangle_{m n}\right)\right) \cup\left(\bigcup_{n=1}^{q}\left(\bigcup_{m=1}^{\infty} \triangle_{m n}\right)\right)
$$

But $\triangle_{p+1, q+1} \subset K$, therefore $x_{m_{j} n_{j}}=\left(\frac{1}{(p+1)(q+1)}, 0\right)>0$ for infinitely many $\left(m_{j}, n_{j}\right)$ 's from $K$ which contradicts $\mathcal{F}-\lim _{j} x_{m_{j} n_{j}}=0$. Therefore, the assumption $\mathcal{I}^{*}-\lim _{m, n} x_{m n}=0$ leads to the contradiction. Hence the converse of the Theorem 3.7 need not be true.

Now the question arises under what condition the converse may hold. The following theorem shows that the converse holds if the ideal $\mathcal{I}$ satisfies the condition (AP).

An admissible ideal $\mathcal{I} \subset P(\mathbb{N} \times \mathbb{N})$ is said to satisfy the condition (AP) if, for every sequence $\left(A_{n}\right)_{n \in \mathbb{N}}$ of pairwise disjoint sets from $\mathcal{I}$, there are sets $B_{n} \subset \mathbb{N}, n \in \mathbb{N}$ such that the symmetric difference $A_{n} \triangle B_{n}$ is a finite set for every $n$ and $\bigcup_{n \in \mathbb{N}} B_{n} \in \mathcal{I}$.

Theorem 3.9 Suppose the ideal $\mathcal{I}$ satisfies the condition $(A P)$. If $x=\left(x_{j k}\right)$ is a double sequence in $X$ such that $\mathcal{I}_{\mathcal{F}}-\lim x=\ell$, then $\mathcal{I}_{\mathcal{F}}^{*} \lim x=\ell$.

Proof Suppose $\mathcal{I}$ satisfies the condition $(\mathrm{AP})$ and $\mathcal{I}_{\mathcal{F}}-\lim x=\ell$. Then, for each $\epsilon>0, t>0$ and nonzero $z \in X$, we have

$$
\left\{(j, k) \in \mathbb{N} \times \mathbb{N}: \mathcal{F}_{x_{j k}-\ell, z}(t) \leq 1-\epsilon\right\} \in \mathcal{I}
$$

We define the set $A_{p}$ for $p \in \mathbb{N}, t>0$ and $z \in X$ as

$$
A_{p}=\left\{(j, k) \in \mathbb{N} \times \mathbb{N}: 1-\frac{1}{p} \leq \mathcal{F}_{x_{j k}-\ell, z}(t)<1-\frac{1}{p+1}\right\} .
$$

Obviously, $\left\{A_{1}, A_{2}, \ldots\right\}$ is countable and belongs to $\mathcal{I}$, and $A_{i} \cap A_{j}=\emptyset$ for $i \neq j$. By the condition (AP), there is a countable family of sets $\left\{B_{1}, B_{2}, \ldots\right\} \in \mathcal{I}$ such that the symmetric difference $A_{i} \triangle B_{i}$ is a finite set for each $i \in \mathbb{N}$ and $B=\bigcup_{i=1}^{\infty} B_{i} \in \mathcal{I}$. From the definition of the associated filter $F(\mathcal{I})$ there is a set $K \in F(\mathcal{I})$ such that $K=\mathbb{N} \times \mathbb{N} \backslash B$. To prove the theorem it is sufficient to show that the subsequence $\left(x_{j k}\right)_{(j, k) \in K}$ is convergent to $\ell$ in $(X, \mathcal{F}, *)$. Let $s>0$ and $t>0$. Choose $q \in \mathbb{N}$ such that $\frac{1}{q}<s$. Then

$$
\left\{(j, k) \in \mathbb{N} \times \mathbb{N}: \mathcal{F}_{x_{j k}-\ell, z}(t) \leq 1-s\right\} \subset\left\{(j, k) \in \mathbb{N} \times \mathbb{N}: \mathcal{F}_{x_{j k}-\ell, z}(t) \leq 1-\frac{1}{q}\right\} \subset \bigcup_{i=1}^{q+1} A_{i}
$$

Since $A_{i} \triangle B_{i}, i=1,2, \ldots, q+1$ are finite, there exists $\left(j_{0}, k_{0}\right) \in \mathbb{N} \times \mathbb{N}$ such that

$$
\left(\bigcup_{i=1}^{q+1} B_{i}\right) \cap\left\{(j, k): j \geq j_{0} \text { and } k \geq k_{0}\right\}=\left(\bigcup_{i=1}^{q+1} A_{i}\right) \cap\left\{(j, k): j \geq j_{0} \text { and } k \geq k_{0}\right\} .
$$


If $j \geq j_{0}, k \geq k_{0}$ and $(j, k) \in K$, then $(j, k) \notin \bigcup_{i=1}^{q+1} B_{i}$. Therefore by (3.1), we have $(j, k) \notin$ $\bigcup_{i=1}^{q+1} A_{i}$. Hence, for every $j \geq j_{0}, k \geq k_{0}$ and $(j, k) \in K$, we have $\mathcal{F}_{x_{j k}-\ell, z}(t)>1-s$. Since $s$ was arbitrary, we have $\mathcal{I}_{\mathcal{F}}^{*} \lim x=\ell$.

This completes the proof of the theorem.

\section{Competing interests}

The authors declare that they have no competing interests.

\section{Authors' contributions}

The authors contributed equally and significantly in writing this paper. Both the authors read and approved the final manuscript.

\section{Received: 12 March 2012 Accepted: 9 August 2012 Published: 31 August 2012}

\section{References}

1. Menger, K: Statistical metrics. Proc. Natl. Acad. Sci. USA 28, 535-537 (1942)

2. Schweizer, B, Sklar, A: Statistical metric spaces. Pac. J. Math. 10, 313-334 (1960)

3. Šerstnev, AN: On the notion of a random normed space. Dokl. Akad. Nauk SSSR 149, 280-283 (1963)

4. Alsina, C, Schweizer, B, Sklar, A: Continuity properties of probabilistic norms. J. Math. Anal. Appl. 208(2), 446-452 (1997)

5. Goleț, I: On probabilistic 2-normed spaces. Novi Sad J. Math. 35(1), 95-102 (2006)

6. Gähler, S: 2-metrische Räume und ihre topologeische Struktur. Math. Nachr. 26, 115-148 (1963)

7. Fast, H: Sur la convergence statistique. Colloq. Math. 2, 241-244 (1951)

8. Çolak, R, Bektaş, CA: $\lambda$-statistical convergence of order $\alpha$. Acta Math. Sci., Ser. B 31(3), 953-959 (2011)

9. Fridy, JA: On statistical convergence. Analysis 5, 301-313 (1985)

10. Karakaya, V: Some geometric properties of sequence spaces involving lacunary sequence. J. Inequal. Appl. 2007, Article ID 81028 (2007)

11. Mohiuddine, SA, Danish Lohani, QM: On generalized statistical convergence in intuitionistic fuzzy normed space. Chaos Solitons Fractals 42, 1731-1737 (2009)

12. Mohiuddine, SA, Şevli, H, Cancan, M: Statistical convergence in fuzzy 2-normed space. J. Comput. Anal. Appl. 12(4), 787-798 (2010)

13. Mohiuddine, SA, Aiyub, M: Lacunary statistical convergence in random 2-normed spaces. Appl. Math. Inf. Sci. 6(3), 581-585 (2012)

14. Mohiuddine, SA, Alotaibi, A, Mursaleen, M: Statistical convergence of double sequences in locally solid Riesz spaces. Abstr. Appl. Anal. 2012, Article ID 719729 (2012)

15. Mohiuddine, SA, Savaș, E: Lacunary statistically convergent double sequences in probabilistic normed spaces. Ann. Univ. Ferrara (2012). doi:10.1007/s11565-012-0157-5

16. Moricz, F: Statistical convergence of multiple sequences. Arch. Math. 81, 82-89 (2003)

17. Mursaleen, M, Edely, OHH: Statistical convergence of double sequences. J. Math. Anal. Appl. 288, $223-231$ (2003)

18. Mursaleen, M, Mohiuddine, SA: Statistical convergence of double sequences in intuitionistic fuzzy normed spaces. Chaos Solitons Fractals 41, 2414-2421 (2009)

19. Mursaleen, M, Mohiuddine, SA: On lacunary statistical convergence with respect to the intuitionistic fuzzy normed space. J. Comput. Appl. Math. 233, 142-149 (2009)

20. Mursaleen, M, Çakan, C, Mohiuddine, SA, Savaş, E: Generalized statistical convergence and core of double sequences. Acta Math. Sin. Engl. Ser. 26(9), 2131-2144 (2010)

21. Savaş, $E$, Mohiuddine, SA: $\bar{\lambda}$-statistically convergent double sequences in probabilistic normed spaces. Math. Slovaca 62(1), 99-108 (2012)

22. Kastyrko, P, Šalát, T, Wilczyński, W: I-convergence. Real Anal. Exch. 26, 669-686 (2000-2001)

23. Das, P, Kastyrko, P, Wilczyński, W, Malik, P: I and I'-convergence of double sequences. Math. Slovaca 58(5), 605-620 (2008)

24. Mursaleen, $\mathrm{M}$, Mohiuddine, $\mathrm{SA}$, Edely, $\mathrm{OHH}$ : On the ideal convergence of double sequences in intuitionistic fuzzy normed spaces. Comput. Math. Appl. 59,603-611 (2010)

25. Mursaleen, M, Mohiuddine, SA: On ideal convergence of double sequences in probabilistic normed spaces. Math. Rep. 12(4), 359-371 (2010)

26. Mursaleen, M, Alotaibi, A: On I-convergence in random 2-normed spaces. Math. Slovaca 61(6), 933-940 (2011)

27. Mursaleen, M, Mohiuddine, SA: On ideal convergence in probabilistic normed spaces. Math. Slovaca 62(1), 49-62 (2012)

28. Sahiner, A, Gürdal, M, Saltan, S, Gunawan, H: Ideal convergence in 2-normed spaces. Taiwan. J. Math. 11(5), 1477-1484 (2007)

29. Gürdal, M, Acik, I: On I-Cauchy sequences in 2-normed spaces. Math. Inequal. Appl. 11(2), 349-354 (2008)

30. Gürdal, M, Pehlivan, S: The statistical convergence in 2-Banach spaces. Thai J. Math. 2(1), 107-113 (2004)

31. Alotaibi, A, Mohiuddine, SA: On the stability of a cubic functional equation in random 2-normed spaces. Adv. Differ. Equ. 2012, 39 (2012)

32. Pringsheim, A: Zur theorie der zweifach unendlichen Zahlenfolgen. Math. Z. 53, 289-321 (1900)

doi:10.1186/1687-1847-2012-149

Cite this article as: Mohiuddine et al.: Ideal convergence of double sequences in random 2-normed spaces. Advances in Difference Equations 2012 2012:149. 